(c) American Dairy Science Association, 2005.

\title{
Analysis of Phospho- and Sphingolipids in Dairy Products by a New HPLC Method
}

\author{
R. Rombaut, J. V. Camp, and K. Dewettinck \\ Ghent University, Faculty of Bioscience Engineering Department of Food Safety \\ and Food Quality (LA07), 9000 Gent, Belgium
}

\begin{abstract}
Dairy phospho- and sphingolipids are gaining interest due to their nutritional and technological properties. A new HPLC method, using an evaporative laser lightscattering detector, was developed, which enabled excellent separation of glucosylceramide, lactosylceramide, phosphatidic acid, phosphatidylethanolamine, phosphatidylinositol, phosphatidylserine, phosphatidylcholine, sphingomyelin, and lysophosphatidylcholine in less than $21 \mathrm{~min}$, including the regeneration of the column. No loss of column performance was observed after 1500 runs because an acid buffer was used. The output signal of the evaporative laser light scattering detector was highly dependent of the flow of the carrier gas and the temperature of the nebulizer, and was maximized by means of a response surface experimental design. Finally, raw milk, cream, butter, buttermilk, Cheddar whey, quarg, and Cheddar cheese were analyzed for their polar lipid content. The absolute values varied substantially ( 0.018 to $0.181 \mathrm{~g} / 100 \mathrm{~g}$ of product). Significant differences were found in the relative content of each polar lipid class among the analyzed products.
\end{abstract}

(Key words: evaporative laser light scattering detection, high-performance liquid chromatography, phospholipid, sphingolipid)

Abbreviation key: ELLSD = evaporative laser lightscattering detector, GLUCER = glucosylceramide, LACCER = lactosylceramide, $\mathbf{L P C}=$ lysophosphatidyl choline, $\mathbf{P A}=$ phosphatidic acid, $\mathbf{P C}=$ phosphatidylcholine, $\mathbf{P E}=$ phosphatidylethanolamine, $\mathbf{P I}=$ phosphatidylinositol, $\mathbf{P L}=$ polar lipids, $\mathbf{P S}=$ phosphatidylserine, SM $=$ sphingomyelin.

\section{INTRODUCTION}

Phospho- and sphingolipids are amphiphilic molecules with lipophilic acyl chains and a hydrophilic head.

Received July 16, 2004.

Accepted October 12, 2004.

Corresponding author: R. Rombaut; e-mail: roeland.rombaut@ ugent.be.
The phospholipids contain a phosphate residue onto which different organic groups may be linked. Sphingolipids can contain a similar organophosphate group or a mono- or disaccharide (glycosphingolipids). In dairy products, important phospholipids are phosphatidylethanolamine (PE), phosphatidylinositol (PI), phosphatidylserine (PS), and phosphatidylcholine (PC). Important sphingolipids in dairy products are sphingomyelin (SM), glucosylceramide (GLUCER), and lactosylceramide (LACCER). Lysophosphatidylcholine (LPC) and phosphatidic acid (PA) are rarely reported in dairy products; when they do appear, it is likely due to careless sample preparation or to phospholipase activity (Christie et al., 1987). In recent years, phosphoand sphingolipids have received renewed interest because of the positive biological activities they exert in the human body. In particular, their ability to reduce blood cholesterol levels (Eckhardt et al., 2002) and to enhance brain functioning (Pepeu et al., 1996), their antioxidative properties (Saito and Ishihara, 1997), their bacteriostatic properties (Sprong et al., 2002), and the inhibitory effect of sphingolipids on colon cancer have been studied intensively (Vesper et al., 1999). Moreover, polar lipids (PL) are extensively used for their functionality and emulsifying qualities in several food systems (Gunstone, 2001; Schneider, 2001).

Dairy products are a good source of these PL (Vesper et al., 1999). The biological membrane of native milk fat globules consists of about one-third phospho- and sphingolipids, stabilizing the milk fat globules in the serum phase of the milk. Analysis of these lipids can be accomplished by means of ${ }^{31} \mathrm{P}$-nuclear magnetic resonance, HPLC, TLC, Fourier transform infrared, and by measuring total phosphorous content (Vanhoutte et al., 2004).

Over the course of the past few decades, HPLC has become the preferred method for the determination of $\mathrm{PL}$, as quantitative and qualitative analysis can readily be obtained at a relatively low cost compared with ${ }^{31} \mathrm{P}$ nuclear magnetic resonance.

Critical points in the analysis of PL in food products are the method of extraction, separation, and detection. Often, little attention is given to the first of these. The majority of PL in food products are present in membra- 
nous structures, interacting with compounds of a complex food matrix, making them difficult to extract. Standard methods of extraction like Röse-Gottlieb and Werner-Schmidt are used extensively for dairy products. Because these methods use a base or acid in combination with heat, they can lead to oxidation and hydrolysis of PL. Therefore cold-extraction procedures like those of Folch et al. (1957) and Bligh and Dyer (1959) using chloroform-methanol or like Hara and Radin (1978), using hexane-isopropanol should be used. After extraction, often a tedious purification or fractionation step using solid phase extraction cartridges or column chromatography is applied. However, the combination of an inadequate extraction procedure and purification step can result in low and variable recoveries and thus in unreliable and inaccurate results.

Several HPLC methods are described for the separation of lecithin and derivatives (Mounts et al., 1992; Abidi et al., 1996; Carelli et al., 1997; Bonekamp and Fiebig, 1999). However, these methods are less applicable to dairy products. Phosphatidylserine, which is only present in trace amounts in lecithin fractions, is often poorly separated from other PL. Moreover, these methods do not consider the presence of SM and cerebrosides.

Most of the recent chromatographic methods used for the separation of PL in dairy products are based on the method of Becart (1990), using a gradient mixture of chloroform, methanol, and a buffer at high $\mathrm{pH}(>7)$ with an alkali modifier like triethylamine or ammonium hydroxide on a plain silica column (Becart et al., 1990; Vaghela and Kilara, 1995; Caboni et al., 1996). The modifier is used to enhance peak shape and resolution. Although enabling a fair separation of most of the PL, the high $\mathrm{pH}$ quickly dissolves the silica packing, thereby seriously reducing column life.

For the chromatographic analysis of fats and oils, the use of evaporative light scattering detection is generally preferred. In this type of detector, the elution solvent from the column is nebulized by the aid of a pressurized gas (compressed air, helium, or nitrogen) in a heating tube. The analyte is not evaporated and passes as an aerosol through a beam of conventional or laser light, which is reflected and refracted. The scattered light is detected by a photomultiplier or a photodiode, which is placed at a fixed angle and is directly related with the quantity of the analyte and the droplet size. The evaporative light-scattering detector is a universal detector that responds to any analyte that is less volatile than the mobile phase. It has a low background signal, is compatible with a broad range of solvents, it allows gradient elution (unlike the refractive index detector), and the signal is independent of the degree of saturation and chain length of an acyl chain (unlike the UV detector). However, the droplet size (and thus the response) is highly dependent on the flow of the nebulizing gas, the temperature of the evaporating tube and the flow rate, and on the composition and physical characteristics of the mobile phase. Therefore, the working conditions should be optimized to ensure the highest possible detector sensitivity and should be reproduced rigorously each time. Otherwise, a recalibration is indispensable when working quantitatively. The mobile phase should be of the highest quality, as nonvolatile impurities would result in an increased background signal, and could alter analyte droplet formation and consequently detector sensitivity. In the last decade, detectors equipped with a laser as a light source came commercially available. These evaporative laser light scattering detectors (ELLSD) outperform other evaporative light-scattering detector models in sensitivity, stability, and reproducibility over longer periods of analysis. After all, compared with conventional light sources, a laser light source is characterized by higher intensity, increased life span, no decline in light intensity over longer periods, minimal equilibration time, and minimal variation between source to source (Onken and Berger, 1998; Koropchak et al., 2000).

This study was performed to develop an HPLCELLSD method, enabling a quick separation of the most abundant PL classes present in dairy products, by means of an acid modifier, without the need of a purification/fractionation step.

\section{MATERIALS AND METHODS}

\section{Materials}

Acid whey and Cheddar whey were obtained from a local dairy plant (Büllinger Butterei, Büllingen, Belgium and Belgomilk, Langemark, Belgium, respectively). Raw milk was obtained from a local farmer. Skimmed milk, buttermilk, Gouda cheese, quarg, butter, and cream samples were obtained from the local supermarket. All samples were stored at $<4^{\circ} \mathrm{C}$ prior to analysis.

Chloroform, hexane, isopropanol and methanol used for extraction were of 99+ grade and obtained from Chem-Lab NV (Zedelgem, Belgium).

Chloroform, methanol, and formic acid used as mobile phase were of HPLC grade and obtained from Acros Organics (Geel, Belgium). Triethylamine (HPLC grade) and PL standards of GLUCER, LACCER, PA, PE, PI, PS, PC, SM, and LPC were obtained from Sigma-Aldrich NV (Bornem, Belgium).

\section{Chromatographic Analysis}

Polar lipid separations were performed on a Thermo Finnigan Surveyor HPLC system with 4 solvent lines, 
a degasser, autosampler, and Atlas 2003 software (Thermo Electron Corp., Brussels, Belgium), which was coupled with an Alltech ELSD 2000 ELLSD (Alltech Associates Inc., Lokeren, Belgium). As the nebulizing gas, $\mathrm{N}_{2}$ was used at a flow rate of $1.4 \mathrm{~L} / \mathrm{min}$, and a nebulizing temperature of $85^{\circ} \mathrm{C}$. The gain was set at 2 and the impactor, a split option for the detection of semivolatiles in combination with aqueous mobile phases, was disabled. The suction head of the chloroform solvent line was replaced by a Hastalloy $\mathrm{C}$ suction head, to prevent phantom peaks during gradient elution.

A $150 \times 3.2 \mathrm{~mm}$ Prevail silica column with $3-\mu \mathrm{m}$ particle diameter (Alltech Associates Inc.) was used. A precolumn with the same packing and internal diameter was used. The elution program was a linear gradient with 87.5:12:0.5 ( $\mathrm{vol} / \mathrm{vol} / \mathrm{vol})$ chloroform:methanol:triethylamine buffer ( $\mathrm{pH} \mathrm{3,1} M$ formic acid) at $\mathrm{t}=0 \mathrm{~min}$ to $28: 60: 12(\mathrm{vol} / \mathrm{vol} / \mathrm{vol})$ at $\mathrm{t}=16 \mathrm{~min}$. The mobile phase was brought back to the initial conditions at $\mathrm{t}=17 \mathrm{~min}$ and the column was allowed to equilibrate until the next injection at $\mathrm{t}=21 \mathrm{~min}$. The flow was maintained at $0.5 \mathrm{~mL} / \mathrm{min}$, which resulted in a backpressure of 55 to 90 bar. The injection volume was $25 \mu \mathrm{L}$. The samples and the column were equilibrated at $40^{\circ} \mathrm{C}$.

\section{Polar Lipid Extraction Procedure}

A modification of the method described by Shaikh (1994) was used to extract the dairy samples. Briefly, $5 \mathrm{~g}$ of sample was diluted with deionized water to 20 $\mathrm{mL}$ and was thoroughly blended with an Ultra-Turrax (model T25B, Ika-Werke, Staufen, Germany). To the slurry, $80 \mathrm{~mL}$ of $2: 1$ ( $\mathrm{vol} / \mathrm{vol}$ ) chloroform:methanol was added and the mixture was transferred into a separatory funnel. After shaking for $2 \mathrm{~min}$, the mixture was allowed to stand and separate and the clear lower chloroform layer was released. This step was repeated twice with $40 \mathrm{~mL}$ of $20: 1$ ( $\mathrm{vol} / \mathrm{vol}$ ) chloroform:methanol that was added to the upper (water) phase. In a fourth step, $40 \mathrm{~mL}$ of $86: 14: 1$ ( $\mathrm{vol} / \mathrm{vol} / \mathrm{vol}$ ) chloroform:methanol:water with $1 \mathrm{~N} \mathrm{HCl}$ and $0.9 \% \mathrm{NaCl}$ was used. This lower phase was released and washed with a $0.9 \% \mathrm{NaCl}$ solution, until neutral $\mathrm{pH}$ was reached. After this, the 4 lower phases were pooled and evaporated, using a rotary vacuum evaporator at $35^{\circ} \mathrm{C}$. The crude lipids were redissolved in exactly $10 \mathrm{~mL}$ of $2: 1$ (vol/vol) chloroform:methanol, transferred into a capped test tube and stored at $-32^{\circ} \mathrm{C}$ until HPLC analysis. Each extraction was performed 3 times, and injected twice.

\section{Statistics}

Statistics were performed with Sigmaplot 6.0 and SPSS 11.5 (SPSS, Inc., Chicago, IL).

\section{RESULTS AND DISCUSSION}

\section{Chromatographic Analysis}

Typical chromatograms of a standard mixture and samples of acid whey, Gouda cheese, and butter are shown in Figure 1. The proposed method is able to separate GLUCER, LACCER, PA, PE, PI, PS, PC, SM, and LPC, even in difficult matrices like cheese and butter, and this in $21 \mathrm{~min}$, including the regeneration of the column. The SM resulted in 2 distinct peaks, as described by others (Christie et al., 1987; Becart et al., 1990; Vaghela and Kilara, 1995). Other peaks are odd shaped and often split in subpeaks, presumably due to the heterogeneity of SM fatty acid residues (Breton et al., 1989). A small drift of retention time was often observed ( $\pm 0.8 \mathrm{~min})$ over longer periods of analysis.

In butter, where the PL content based on total fat is as low as $0.25 \%$, no unacceptable interference of poorly retained compounds (mono-, di-, and triglycerides, FFA, sterols, ceramides, etc.) could be observed. Therefore, removal of the apolar fraction in a preceding clean-up step with solid phase extraction cartridges is not required.

The use of a buffer is necessary to increase the peak shape and resolution of PS and PI. At $\mathrm{pH} 2$, PS and PI co-elute. At $\mathrm{pH} 4$, the resolution between $\mathrm{PS}$ and $\mathrm{PC}$ is decreased, and at $\mathrm{pH} 5, \mathrm{PS}$ and PC fuse together. By using an acidic buffer, the column life was considerably extended compared with the use of a buffer at $\mathrm{pH}>7$. More than 1500 runs could be performed without loss of column performance. The use of a 3.2-mm i.d. column decreased the solvent use with $48 \%$, compared with a normal $4.6-\mathrm{mm}$ i.d.

Figure 2 shows the calibration curves for PC, SM, and LACCER. All other curves are situated between the curves of PC and SM. A linear response was found for all PL classes. In Table 1, the slope, intercept, $R^{2}$, and the observed range of each calibration curve is given. All curves were visually compared. The ELLSD is believed to be a uniform mass-sensitive detector, but the signal is dependent on each PL class, as there was no overlap of the $95 \%$ confidence intervals, except for PS and LPC. This conclusion can also be drawn from the slope and intercept in Table 1, taking the variation into account. Although the theoretical response of an evaporative light scattering detector is sigmoidal, a broad linear working range ( $>1$ log unit) can be defined for each PL. The intercept is substantial and negative for each PL due to the nonlinear response at very low concentrations. The detectability limit of each component was defined as the average of concentrations of noise area just before and after each peak of 10 equivalent whey samples +6 times the standard deviation of these noise areas. These values are also shown in Table 

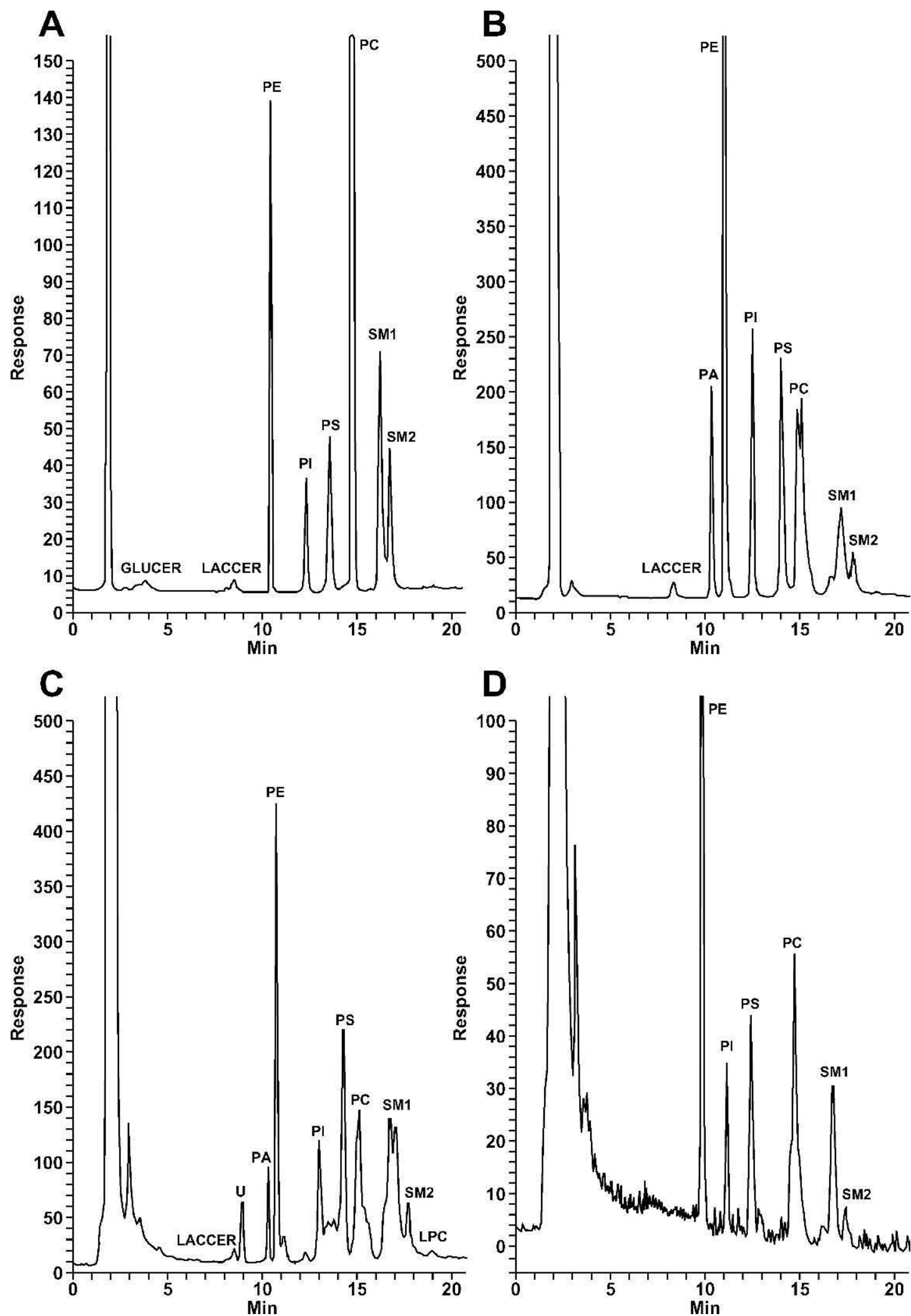

Figure 1. High-performance liquid chromatography chromatograms of A) polar lipid standards, B) acid whey, C) Gouda cheese, D) butter. $\mathrm{U}=$ unknown, GLUCER = glucosylceramide, LACCER = lactosylceramide, LPC = lysophosphatidylcholine, PA = phosphatidic acid, $\mathrm{PC}=$ phosphatidylcholine, $\mathrm{PE}=$ phosphatidylethanolamine, $\mathrm{PI}=$ phosphatidylinositol, $\mathrm{PS}=$ phosphatidylserine, $\mathrm{SM}=\mathrm{sphingomyelin}$. 


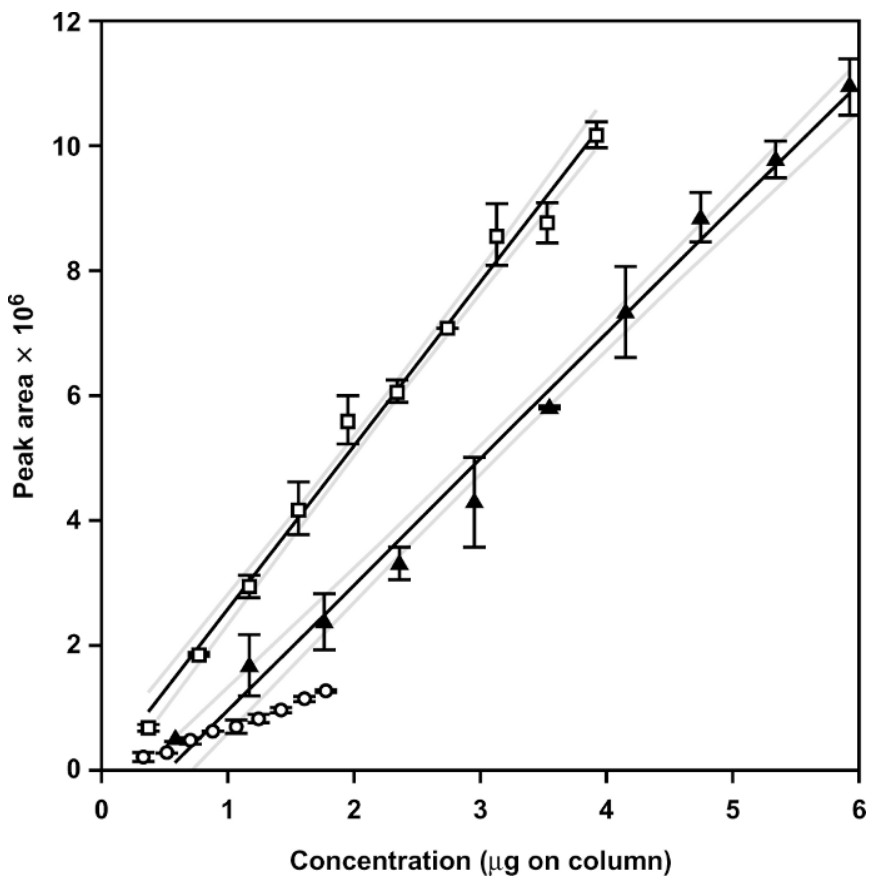

Figure 2. Calibration curves for PC $(\square)$, SM ( $\Delta$ ), and LACCER $(\bigcirc)$. Each point is represented as the average of 2 measurements with the standard error as error bars. Regression lines were calculated on the original points. The gray lines represent the $95 \%$ confidence interval of each regression line. LACCER = lactosylceramide, PC = phosphatidylcholine, $\mathrm{SM}=$ sphingomyelin.

1 and are in line ( 11 to $40 \mathrm{ng}$ ) with other authors' results (Becart et al., 1990).

\section{Optimization of Detector Settings}

The optimal settings of the ELLSD (flow of the nebulizing gas and temperature) were determined by means of a response surface experimental design. Design maxima $\left(\mathrm{T}_{\max }=110^{\circ} \mathrm{C}\right.$ and Flow $\left.{ }_{\max }=3.5 \mathrm{~L} / \mathrm{min}\right)$ were de-

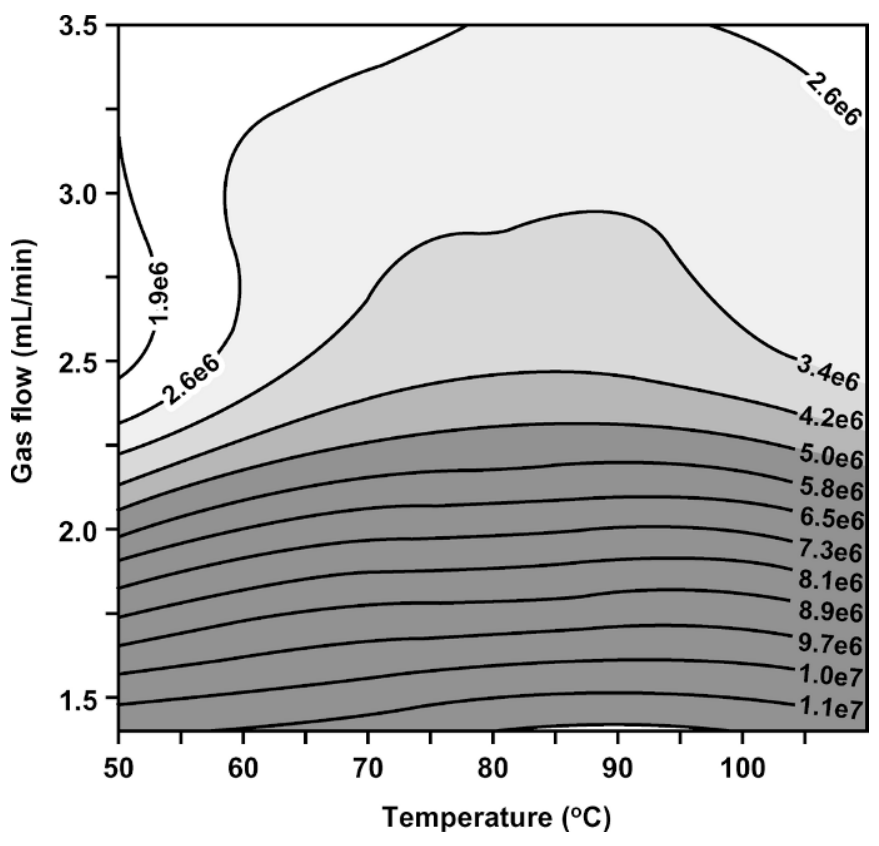

Figure 3. Detector output for phosphatidylcholine in function of temperature of the nebulizer and flow of the nebulizing gas $\left(\mathrm{N}_{2}\right)$.

fined as the limits of the detector itself. Minima $\left(\mathrm{T}_{\min }=\right.$ $50^{\circ} \mathrm{C}$ and Flow $_{\min }=1.4 \mathrm{~L} / \mathrm{min}$ ) are those values above which no change in baseline noise could be observed. For each combination (13 in total, 5 at the center point), a full chromatogram of a solution of milk PL was recorded. Peak area of each PL was chosen as response value.

Analysis of variance revealed that a quadratic model was highly significant for each PL. The $P$ and $R^{2}$ values ranged from $P<0.0005$ and $\mathrm{R}^{2}=0.935$ (GLUCER) to $P<0.0001$ and $\mathrm{R}^{2}=0.992(\mathrm{PI})$, respectively. Lack-offit tests were all nonsignificant, and no outliers were observed. In Figure 3, the response is given for PC.

Table 1. Slope ( $\pm \mathrm{SE})$, intercept $( \pm \mathrm{SE}), \mathrm{R}^{2}$, linearity range, and detectability limit of calibration curves of polar lipid classes.

\begin{tabular}{llllll}
\hline $\begin{array}{l}\text { Polar lipid } \\
\text { lass }^{1}\end{array}$ & Slope $\left(\times 10^{6}\right)$ & Intercept $\left(\times 10^{5}\right)$ & $\mathrm{R}^{2}$ & $\begin{array}{l}\text { Range } \\
(\mu \mathrm{g} \text { on column })\end{array}$ & $\begin{array}{l}\text { Detectability } \\
\text { limit } \\
(\mu \mathrm{g} \text { on column })\end{array}$ \\
\hline GLUCER & $1.09 \pm 0.03$ & $-0.57 \pm 0.10$ & 0.989 & 0.05 to 0.5 & 0.027 \\
LACCER & $0.73 \pm 0.03$ & $-1.00 \pm 0.31$ & 0.993 & 0.35 to 1.8 & 0.040 \\
PA & $1.39 \pm 0.03$ & $-2.55 \pm 0.39$ & 0.995 & 0.25 to 2.5 & 0.021 \\
PE & $2.50 \pm 0.07$ & $-7.61 \pm 1.32$ & 0.988 & 0.30 to 3.0 & 0.012 \\
PI & $2.10 \pm 0.10$ & $-4.71 \pm 0.96$ & 0.969 & 0.16 to 1.6 & 0.014 \\
PS & $1.63 \pm 0.04$ & $-2.48 \pm 0.55$ & 0.993 & 0.21 to 2.1 & 0.018 \\
PC & $2.63 \pm 0.07$ & $-1.39 \pm 1.73$ & 0.992 & 0.39 to 3.9 & 0.011 \\
SM & $2.01 \pm 0.06$ & $-11.07 \pm 2.28$ & 0.991 & 0.59 to 5.9 & 0.014 \\
LPC & $1.67 \pm 0.05$ & $-3.81 \pm 0.71$ & 0.988 & 0.24 to 2.4 & 0.017 \\
\hline
\end{tabular}

${ }^{1}$ GLUCER = Glucosylceramide; LACCER = lactosylceramide; PA = phosphatidic acid; PE = phosphatidylethandamine; $\mathrm{PI}=$ phosphatidylinositol $; \mathrm{PS}=$ phosphatidylserine; $\mathrm{PC}=$ phosphatidylcholine; $\mathrm{SM}=$ sphingomyelin; LPC = lysophosphatidylcholine. 
The response was highly dependent on the flow of the nebulizer gas $(P<0.0001)$, decreasing sharply with increasing flow, and this was true for all PL. The effect of temperature was less pronounced, and varied depending on the type of PL. A maximum response (and corresponding detector settings) could be defined for each PL. As the response of the major PL is of less significance than the minor ones, the optimal detector settings were determined by using a desirability function as described by Derringer and Suich (1980), stating a maximum response for the minor PL (i.e., GLUCER, LACCER, PI, and PS). As such, maximal desirability was found at $\mathrm{T}_{\text {opt }}=85^{\circ} \mathrm{C}$ and Flow $_{\text {opt }}=1.4 \mathrm{~L} / \mathrm{min}$.

\section{Analysis of Dairy Samples}

Raw milk, cream, butter, buttermilk, Cheddar whey, skimmed quarg, and Cheddar cheese were analyzed on PL content. The results are given in Table 2 and are the average of 4 values. These contents are in agreement with other authors' observations (Christie et al., 1987; Vesper et al., 1999). Phosphatidic acid and LPC were not observed in detectable amounts. The sum of GLUCER, LACCER, PE, PI, PS, PC and SM is regarded as total PL. Fat-rich products are high in PL, as they are situated in the milk fat globule membrane, which surrounds the fat globule. However, during churning, the milk fat globule membrane is ruptured, and migrates substantially toward the water phase (Michalski et al., 2002), resulting in a low-fat product (buttermilk) rich in PL. This can be observed when the PL content is expressed based on total fat content. Onefifth of buttermilk lipids are PL. When expressing the PL content on a DM basis, all samples are in the same range, except for buttermilk, the PL content of which is 4 to 5 times higher than for the other samples. As such, buttermilk could be an interesting source for further purification of the PL to create a product with a high technological and functional value.

Analysis of variance revealed a significant difference in relative composition of PL classes among the dairy samples $(P<0.05)$. Phosphatidylethanolamine is by far the most predominant PL (36.5 to 42.9\%), followed by PC (18.7 to $20.7 \%$ ), and SM (12.8 to $20.2 \%$ ). These 3 species represent more than $70 \%$ of total PL in dairy samples. The differences in relative amount can be ascribed to a preferential enrichment of certain classes during processing, or to differences in the original raw milk. More research should be conducted in this field.

\section{ACKNOWLEDGMENTS}

The authors would like to thank Büllinger Bütterei and Belgomilk Langemark for their kind donation of

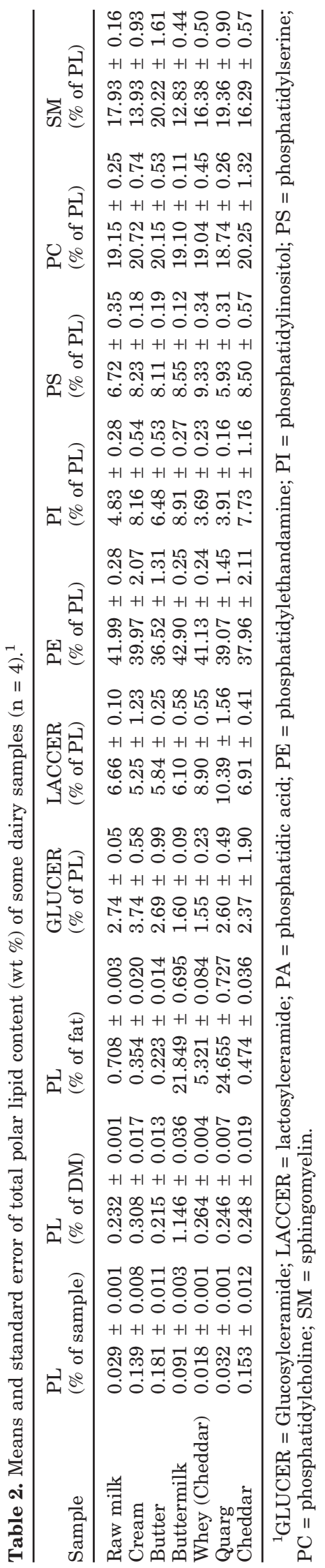

Journal of Dairy Science Vol. 88, No. 2, 2005 
the acid and sweet whey, respectively, and B. Lewille and M. Jooris for their technical assistance.

\section{REFERENCES}

Abidi, S. L., T. L. Mounts, and T. Finn. 1996. A preferred solvent system for high-performance liquid chromatographic analysis of soybean phospholipids with evaporative light-scattering detection. J. AOCS 73:535-536.

Becart, J., C. Chevalier, and J. Biesse. 1990. Quantitative analysis of phospholipids by HPLC with a light-scattering evaporating detector-Application to raw materials for cosmetic use. J. High Resolut. Chromatogr. 13:126-129.

Bligh, E. G., and W. J. Dyer. 1959. A rapid method of total lipid extraction and purification. Can. J. Biochem. Physiol. 37:911-917.

Bonekamp, A., and H. J. Fiebig. 1999. Bestimmung der Phospholipide durch HPLC. Fett Lipid 101:80-83.

Breton, L., B. Serkiz, J. P. Volland, and J. Lepagnol. 1989. A new rapid method for phospholipid separation by high-performance liquid-chromatography with light-scattering detection. J. Chromatogr. B. 497:243-249.

Caboni, M. F., S. Menotta, and G. Lercker. 1996. Separation and analysis of phospholipids in different foods with a light-scattering detector. J. AOCS 73:1561-1566.

Carelli, A. A., M. I. V. Brevedan, and G. H. Crapiste. 1997. Quantitative determination of phospholipids in sunflower oil. J. AOCS 74:511-514

Christie, W. W., R. C. Noble, and G. Davies. 1987. Phospholipids in milk and dairy-products. J. Soc. Dairy Technol. 40:10-12.

Derringer, G., and R. Suich. 1980. Simultaneous optimization of several response variables. J. Qual. Technol. 12:214-219.

Eckhardt, E. R. M., D. Q. H. Wang, J. M. Donovan, and M. C. Carey. 2002. Dietary sphingomyelin suppresses intestinal cholesterol absorption by decreasing thermodynamic activity of cholesterol monomers. Gastroenterology 122:948-956.

Folch, J., M. Lees, and G. H. Sloane-Stanley. 1957. A simple method for the isolation and purification of total lipids from animal tissues. J. Biol. Chem. 226:497-509.
Gunstone, F. D. 2001. Structured and Modified Lipids. Marcel Dekker, New York, NY.

Hara, A., and N. S. Radin. 1978. Lipid extraction of tissues with a low-toxicity solvent. Anal. Biochem. 90:420-426.

Koropchak, J. A., L. E. Magnusson, M. Heybroek, S. Sadain, X. H. Yang, and M. P. Anisimov. 2000. Fundamental aspects of aerosolbased light-scattering detectors for separations. Adv. Chromatogr. 40:275-314.

Michalski, M. C., F. Michel, D. Sainmont, and V. Briard. 2002. Apparent zeta-potential as a tool to assess mechanical damages to the milk fat globule membrane. Colloids Surf. B 23:23-30.

Mounts, T. L., S. L. Abidi, and K. A. Rennick. 1992. HPLC analysis of phospholipids by evaporative laser light-scattering detection. J. AOCS 69:438-442.

Onken, J., and R. G. Berger. 1998. Evaporative light scattering detector (ELSD) for the analysis in food chemistry. Dtsch. Lebensm.Rundsch. 94:287-292.

Pepeu, G., I. M. Pepeu, and L. Amaducci. 1996. A review of phosphatidylserine pharmacological and clinical effects. Is phosphatidylserine a drug for the ageing brain? Pharmacol. Res. 33:73-80.

Saito, H., and K. Ishihara. 1997. Antioxidant activity and active sites of phospholipids as antioxidants. J. AOCS 74:1531-1536.

Schneider, M. 2001. Phospholipids for functional food. Eur. J. Lipid Sci. Tech. 103:98-101.

Shaikh, N. A. 1994. Assessment of various techniques for the quantitative extraction of lysophospholipids from myocardial tissues. Anal. Biochem. 216:313-321.

Sprong, R. C., M. F. E. Hulstein, and R. van der Meer. 2002. Bovine milk fat components inhibit food-borne pathogens. Int. Dairy J. 12:209-215

Vaghela, M. N., and A. Kilara. 1995. Quantitative analysis of phospholipids from whey-protein concentrates by high-performance liquid chromatography with a narrow-bore column and an evaporative light-scattering detector. J. AOCS 72:729-733.

Vanhoutte, B., R. Rombaut, K. Dewettinck, and P. Van der Meeren. 2004. Phospholipids. Pages 349-382 in Food Analysis. Vol. 1. L. M. L. Nollet, ed. Marcel Dekker, New York, NY.

Vesper, H., E. M. Schmelz, M. N. Nikolova-Karakashian, D. L. Dillehay, D. V. Lynch, and A. H. Merrill. 1999. Sphingolipids in food and the emerging importance of sphingolipids to nutrition. J. Nutr. 129:1239-1250. 